\title{
Finite temperature Lattice QCD with two flavors of improved Wilson fermions
}

\author{
V.G. Bornyakov ${ }^{* a b}$, S.M. Morozov ${ }^{b}$, Y. Nakamura ${ }^{c f}$, M.I. Polikarpov ${ }^{b}$, \\ G. Schierholz ${ }^{c d}$, and T. Suzuki ${ }^{e f}$ \\ ${ }^{a}$ Institute of High Energy Physics, 142281 Protvino, Russia \\ ${ }^{b}$ Institute for Theoretical and Experimental Physics , B.Cheremushkinskaya 25, 117259 Moscow, \\ Russia \\ ${ }^{c}$ John von Neumann-Institut für Computing NIC / DESY, 15738 Zeuthen, Germany \\ ${ }^{d}$ Deutsches Elektronen-Synchrotron DESY, 22603 Hamburg, Germany \\ ${ }^{e}$ Institute for Theoretical Physics, Kanazawa University, Kanazawa 920-1192, Japan \\ ${ }^{f}$ RIKEN, Radiation Laboratory, Wako, 351-0158, Japan \\ E-mail: Vitaly.Bornyakov@ihep.ru, Yoshifumi.Nakamura@desy.de
}

\section{DIK Collaboration}

We present results of finite temperature QCD from $N_{f}=2$ flavors of non-perturbatively improved Wilson fermions on lattices with $N_{t}=8,10$ and 12. The transition temperature $T_{c}$ is determined for the first time at lattice spacings as low as $a=0.08 \mathrm{fm}$, albeit at pion masses $1.3 \lesssim r_{0} m_{\pi}$. We furthermore compute the screening masses in various color channels at $T>T_{c}$.

The XXV International Symposium on Lattice Field Theory

July 30 - August 4, 2007

Regensburg, Germany

${ }^{*}$ Speaker. 


\section{Introduction}

Despite many efforts to accurately determine the transition temperature $T_{c}$ in finite temperature QCD, the final answer is still open. The problem is that the lattice data need to be extrapolated to the continuum limit and to the physical quark mass. Indeed, recent work of the RBC-Bielefeld [1] and the Wuppertal group [2], using different versions of staggered fermions, report results for $T_{c}$, which differ by more than $10 \%$. (See also the plenary talks of Karsch and Fodor in this volume.) To settle this issue, alternative calculations, preferably using different fermionic actions, are demanded.

In this talk we shall present results for $T_{c}$ from $N_{f}=2$ flavors of nonperturbatively improved Wilson fermions. (For similar work see [3] and the talk of Namekawa in this volume.) Furthermore, we shall report results for the screening mass, which is controversial as well.

We use the plaquette action. The fermionic action reads

$$
S_{F}=S_{F}^{(0)}-\frac{\mathrm{i}}{2} \kappa g c_{s w} a^{5} \sum_{s} \bar{\psi}(s) \sigma_{\mu \nu} F_{\mu v}(s) \psi(s),
$$

where $S_{F}^{(0)}$ is the standard Wilson action, and $c_{S W}$ is determined nonperturbatively [4]. Here we report preliminary results on $24^{3} \times 12$ lattices at $\beta=5.29$, and use our earlier results on $16^{3} \times 8$ at $\beta=5.2,5.25$ and $24^{3} \times 10$ at $\beta=5.2[5,6]$. The transition temperature was determined for lattice spacing $a$ ranging from $0.17 r_{0}$ to $0.23 r_{0}$, and the pion mass $r_{0} m_{\pi}$ varying between 1.3 and 2.7. The lattice spacing and pion mass has been determined from the $T=0$ results obtained by the QCDSF-UKQCD collaboration (see e.g. [7]).

\section{Critical temperature}

We use the Polyakov loop susceptibility $\chi_{L}$ to compute $T_{c}$. In Fig. 1 we show our results on the $24^{3} \times 12$ lattice. The critical value of $\kappa, \kappa_{t}$, is identified as a point, where $\chi_{L}$ reaches its maximum. Applying a Gaussian fit in the vicinity of the maximum we find $\kappa_{t}=0.13589(6)$. Respective value of $T_{c} r_{0}$ is $0.487(6)$

To determine $T_{c}$ in the continuum limit and at the physical pion mass we fit the mass and cut-off dependence by

$$
r_{0} T_{c}\left(r_{0} m_{\pi}, 1 / N_{t}\right)=r_{0} T_{c}(0,0)+c_{N} \cdot \frac{1}{N_{t}^{2}}+c_{m} \cdot\left(r_{0} m_{\pi}\right)^{d}
$$

with $d=1.08$, assuming that the transition is of second order in the chiral limit and is in the universality class of the $3 d O(4)$ spin model. Note that a first order transition at the physical quark masses is not fully excluded [8]. In that case $d=2$. We treat the difference between the two fits with $d=1.08$ and $d=2$ as a systematic error. We also considered an extrapolation of the form

$$
r_{0} T_{c}\left(r_{0} m_{\pi}, a / r_{0}\right)=r_{0} T_{c}(0,0)+c_{a} \cdot\left(\frac{a}{r_{0}}\right)^{2}+c_{m} \cdot\left(r_{0} m_{\pi}\right)^{d} .
$$

Again, the difference between (2.1) and (2.2) was taken as a systematic error.

Our results are presented in Fig. 2, where we show the fit function (2.1) for $N_{t}=8,10,12$ and $N_{t}=\infty$. It turns out that the coefficients $c_{N}$ (respectively $c_{a}$ ) and $c_{m}$ are strongly correlated. 


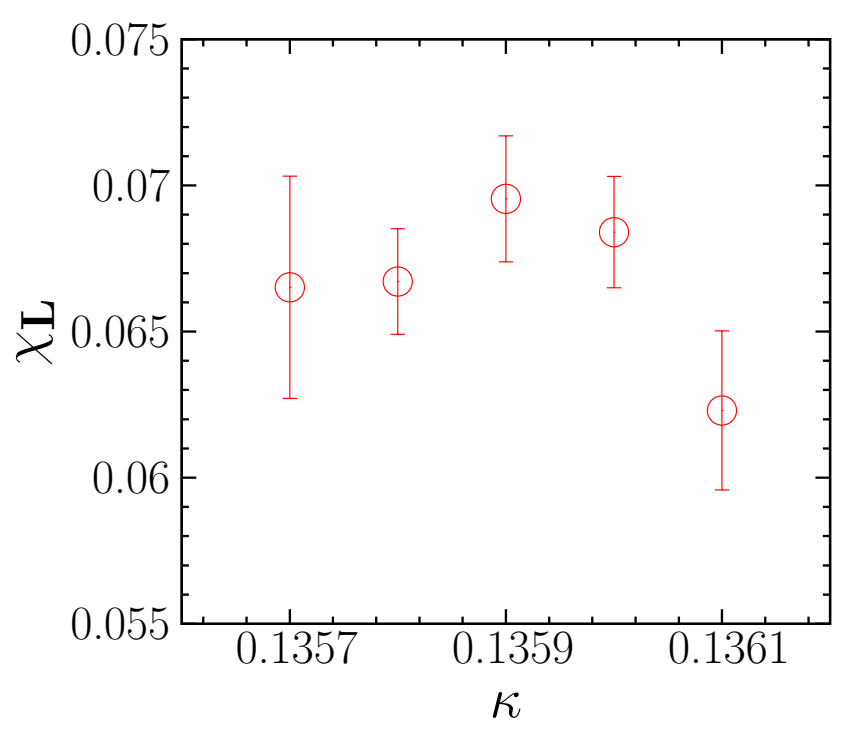

Figure 1: The Polyakov loop susceptibility from the $24^{3} \times 12$ lattice.

The error on (e.g.) $c_{N}$ is approximately $50 \%$, so that the scaling violations might be significantly smaller than displayed in the figure. At present only the result in the physical limit can be trusted entirely. In the continuum limit and at the physical pion mass we obtain

$$
r_{0} T_{c}\left(r_{0} m_{\pi}^{p h}, 0\right)=0.438(6)\left({ }_{-7}^{+13}\right),
$$

where the first error is statistical and the second one systematic.

For comparison we also show the fit function of [1]. It would be consistent with our results (within the error bars) if $c_{N}=0$. The result of our fit (2.1) is $2 \sigma$ away from that value.

As we said already, we used the Polyakov loop susceptibility to compute $T_{c}$. Another observable, the chiral susceptibility, determined from the low lying eigenmodes of the valence overlap Dirac operator, was computed on our $24^{3} \times 10$ lattice in the parallel talk of V. Weinberg in this volume. The preliminary conclusion was that the respective transition temperature is shifted to a lower value as compared to the Polyakov loop susceptibility. This observation is in agreement with the findings of Ref.[2].

\section{Screening masses at $T>T_{c}$}

The study of free energies of static quarks in the high temperature phase is important for understanding of the fate of charmonia above $T_{c}$, as well as for checking the validity of high temperature 


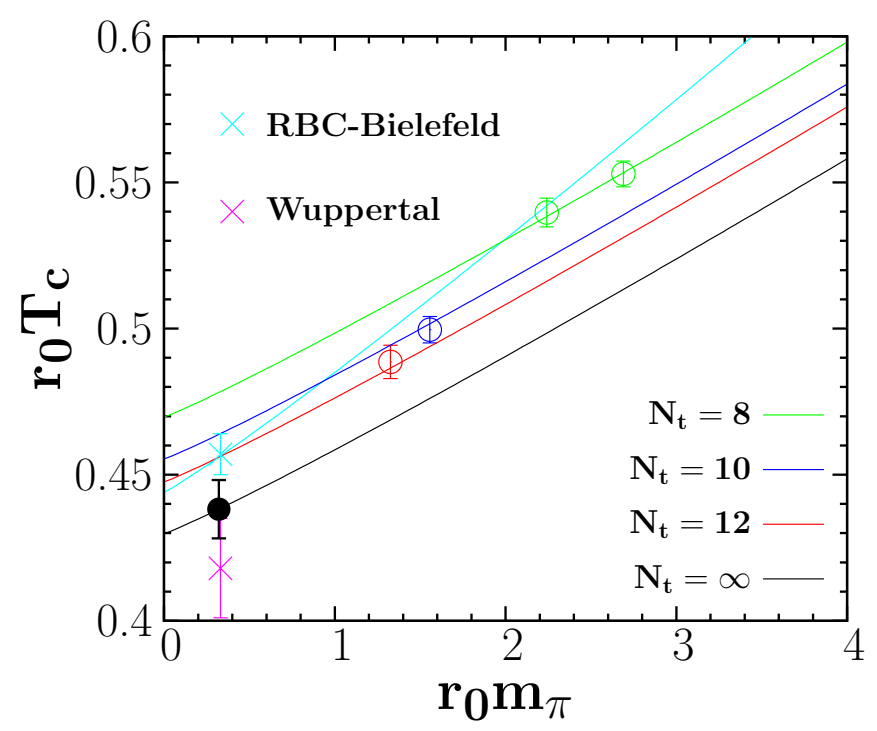

Figure 2: The critical temperature. The solid lines show results of the fit function (2.1). The blue line shows the fit of [1].

perturbation theory. The free energy in the different color channels is given by [9]:

$$
\begin{aligned}
e^{-F_{1}(R, T) / T} & =\frac{1}{3}\left\langle\operatorname{Tr} L^{\dagger}(x) L(y)\right\rangle \\
e^{-F_{8}(R, T) / T} & =\frac{1}{8}\left\langle\operatorname{Tr} L^{\dagger}(x) \operatorname{Tr} L(y)\right\rangle-\frac{1}{24}\left\langle\operatorname{Tr} L^{\dagger}(x) L(y)\right\rangle \\
e^{-F_{6}(R, T) / T} & =\frac{1}{12}\langle\operatorname{Tr} L(x) \operatorname{Tr} L(y)\rangle+\frac{1}{12}\langle\operatorname{Tr} L(x) L(y)\rangle \\
e^{-F_{3}^{*}(R, T) / T} & =\frac{1}{6}\langle\operatorname{Tr} L(x) \operatorname{Tr} L(y)\rangle-\frac{1}{6}\langle\operatorname{Tr} L(x) L(y)\rangle
\end{aligned}
$$

The above definitions are not explicitly gauge invariant. It was argued in Ref. [10] that a gauge invariant result can be obtained after proper gauge fixing. Although a rigorous proof is still lacking and both definitions (3.1) and gauge invariance were questioned in Ref. [11] and Ref. [12], respectively, we, as most of other authors, choose here the Coulomb gauge to study potentials determined by eqs. (3.1). An iterative gauge fixing algorithm with one gauge copy has been applied. It is known that in the Coulomb gauge some gauge noninvariant quantities, e.g. field propagators, suffer from the Gribov problem. To check for the effect of Gribov copies we looked at three copies on a subset of our configurations but found no effect. Still, further, more extensive, checks of these effects are necessary. In particular, a more effective simulated annealing algorithm should be applied.

To improve the signal to noise ratio we used a hypercubic blocking procedure [13]. This reduced the statistical errors by about a factor of three. 
We applied the usual fitting function to describe the static potentials as functions of $R$ at large $R T$ :

$$
V_{i}(R, T) \equiv F_{i}(R, T)-F_{i}(\infty, T)=-C_{i} \frac{\alpha_{i}(T)}{R} e^{-m_{D i}(T) R}
$$

( $i=1,3,6,8)$, where $\alpha_{i}(T)$ and $m_{D i}(T)$ are fit parameters, and $C_{i}$ is the Casimir factor.

Previous studies in $N_{f}=2$ lattice QCD were performed with staggered fermions [14] as well as with improved Wilson fermions [15], in both cases on lattices with $N_{t}=4$. In Ref. [14] results were presented for $m_{\pi} / m_{\rho}=0.7$ and temperatures up to $T / T_{c}=4$. These authors fitted the screening mass $m_{D}$, extracted from the singlet static potential by means of (3.2), in terms of the two-loop perturbative expression times a constant $A$ to allow for nonperturbative corrections:

$$
\frac{m_{D}}{T}=A\left(1+\frac{N_{f}}{6}\right)^{1 / 2} g_{t w o-l o o p}(T) .
$$

They found $A \approx 1.4$, to be compared with $A=1$ in perturbation theory.

In Ref. [15] approximately the same values of $m_{\pi} / m_{\rho}\left(m_{\pi} / m_{\rho}=0.65,0.80\right)$ and same temperature range were explored. The authors confirmed Casimir scaling for $V_{M}(R, T)$, i.e. independence of $\alpha_{i}(T)$ and $m_{D i}(T)$ on $i$. Furthermore, they found the phenomenological relation:

$$
\frac{m_{D}}{T}=\left(1+\frac{N_{f}}{6}\right)^{1 / 2} \sqrt{4 \pi \alpha(T)}
$$

Comparison with results of Ref. [14] revealed agreement for $\alpha(T)$, but $20 \%$ deviation for $m_{D}(T)$. So far the disagreement of $m_{D}(T)$ is unclear. One possible reason is that in both cases lattices with large lattice spacing were used. Thus our result obtained on lattice with small lattice spacing can shed some light on this problem.

Our calculations of the static potentials are performed on $16^{3} \times 8$ lattice at $T / T_{c}=1.27$, the highest available temperature. In Fig. 3 we compare our result for $V_{1}(R, T)$ with that of Ref. [15]. We find good agreement for $R T>0.5$. The disagreement at smaller distances might be partially due to breaking of rotational invariance and HYP smearing of the data of Ref. [15]. Similar agreement was found for other color channels. Thus we confirm the observations made in [15], i.e Casimir scaling and phenomenological scaling (3.4).

\section{Conclusions}

We computed $T_{c}$ for $N_{f}=2$ with improved Wilson fermions on lattices with $N_{t}=8,10,12$ and performed a continuum and chiral extrapolation. The physical value of $r_{0} T_{c}$ was found to be in agreement with both the RBC-Bielefeld and Wuppertal results within the error bars.

The screening masses at $T / T_{c} \approx 1.3$ are found in full agreement with WHOT-QCD results [15], confirming disagreement with the results of staggered fermions [14], as well as Casimir scaling of $V_{i}(R, T)$ and phenomenological scaling of $m_{D}(T)$.

\section{Acknowledgments}

This work is supported by the Large Scale Simulation Program No.07-14-B of High Energy Accelerator Research Organization (KEK). The numerical simulations were done using RSCC at 


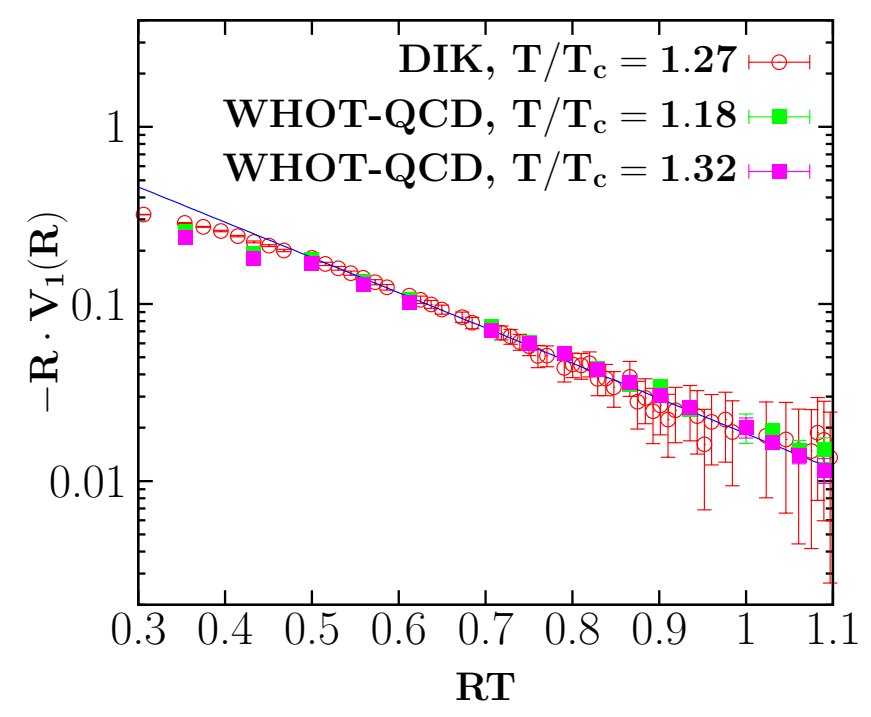

Figure 3: Comparison with [15] for the singlet potential.

RIKEN and BlueGene/L at KEK. V.G.B., S.M.M. and M.I.P. are supported by grants RFBR 0502-16306, 07-02-00237-a, RFBR-DFG 06-02-04010, DFG-RFBR 436 RUS 113/739/0-1 and by the EU Integrated Infrastructure Initiative Hadron Physics (I3HP) under contract RII3-CT-2004506078. S.M.M. is also supported by an INTAS YS fellowship 05-109-4821.

\section{References}

[1] M. Cheng et al., The transition temperature in QCD, Phys. Rev. D 74 (2006) 054507 [arXiv:hep-lat/0608013].

[2] Y. Aoki, Z. Fodor, S. D. Katz and K. K. Szabo, The QCD transition temperature: Results with physical masses in the continuum limit, Phys. Lett. B 643 (2006) 46 [arXiv:hep-lat/0609068].

[3] Y. Maezawa, S. Aoki, S. Ejiri, T. Hatsuda, N. Ishii, K. Kanaya and N. Ukita, Thermodynamics of two-flavor lattice QCD with an improved Wilson quark action at non-zero temperature and density, $\mathrm{J}$. Phys. G 34 (2007) S651 [arXiv:hep-lat/0702005].

[4] K. Jansen and R. Sommer [ALPHA collaboration], $O(\alpha)$ improvement of lattice $Q C D$ with two flavors of Wilson quarks, Nucl. Phys. B 530, 185 (1998) [Erratum-ibid. B 643, 517 (2002)] [hep-lat/9803017].

[5] V. G. Bornyakov et al. [DIK Collaboration], Finite temperature QCD with two flavors of non-perturbatively improved Wilson fermions, Phys. Rev. D 71 (2005) 114504 [arXiv:hep-lat/0401014].

[6] V. G. Bornyakov et al., Critical temperature in QCD with two flavors of dynamical quarks, PoS LAT2005 (2006) 157 [arXiv:hep-lat/0509122]. 
[7] M. Gockeler et al., Simulating at realistic quark masses: Light quark masses, PoS LAT2006 (2006) 160 [arXiv:hep-lat/0610071].

[8] M. D'Elia, A. Di Giacomo and C. Pica, Two flavor QCD and confinement, Phys. Rev. D 72 (2005) 114510 [arXiv:hep-lat/0503030].

[9] L. D. McLerran and B. Svetitsky, Quark Liberation At High Temperature: A Monte Carlo Study Of SU(2) Gauge Theory, Phys. Rev. D 24 (1981) 450.

[10] O. Philipsen, Non-perturbative formulation of the static color octet potential, Phys. Lett. B 535 (2002) 138 [arXiv:hep-lat/0203018].

[11] O. Jahn and O. Philipsen, The Polyakov loop and its relation to static quark potentials and free energies, Phys. Rev. D 70 (2004) 074504 [arXiv:hep-lat/0407042].

[12] V. A. Belavin, V. G. Bornyakov and V. K. Mitrjushkin, On the gauge dependence of the singlet and adjoint potentials, Phys. Lett. B 579 (2004) 109 [arXiv:hep-lat/0310033].

[13] A. Hasenfratz and F. Knechtli, Flavor symmetry and the static potential with hypercubic blocking, Phys. Rev. D 64 (2001) 034504 [arXiv:hep-lat/0103029].

[14] O. Kaczmarek and F. Zantow, Static quark anti-quark interactions in zero and finite temperature QCD. I: Heavy quark free energies, running coupling and quarkonium binding, Phys. Rev. D 71 (2005) 114510 [arXiv:hep-lat/0503017].

[15] Y. Maezawa, N. Ukita, S. Aoki, S. Ejiri, T. Hatsuda, N. Ishii and K. Kanaya [WHOT-QCD Collaboration], Heavy-Quark Free Energy, Debye Mass, and Spatial String Tension at Finite Temperature in Two Flavor Lattice QCD with Wilson Quark Action, Phys. Rev. D 75 (2007) 074501 [arXiv:hep-lat/0702004]. 